\title{
IJCARS: MICCAI 2020 special issue
}

\author{
Anne Martel $^{1} \cdot$ Danail Stoyanov $^{2} \cdot$ Diana Mateus $^{3} \cdot$ Leo Joskowicz $^{4} \cdot$ Purang Abolmaesumi $^{5}$
}

Accepted: 23 September 2021 / Published online: 15 October 2021

(c) CARS 2021

As Guest Editors, we are pleased to present this special issue of IJCARS. It highlights some of the key contributions relating to Image-guided Interventions presented at the 23rd International Conference on Medical Image Computing and Computer-Assisted Intervention (MICCAI 2020).

MICCAI 2020 was held as a virtual meeting, given the highly unusual circumstances imposed globally as a result of the COVID-19 pandemic. Nevertheless, the conference received a total of 1876 full submissions, which were reduced to 1809 submissions following an initial quality control by the program chairs. Of those, $38 \%$ self-declared as either Computer-Assisted Intervention (CAI) or at the intersection of CAI and Medical Imaging Computing (MIC). Following a multistage double-blind review process, managed by 82 area chairs and over 1400 reviewers, $30 \%$ of the papers were accepted and published in the seven volume conference proceedings by Springer Nature.

We used the recommendation of Area Chairs and the review scores and invited the authors of highest ranking papers related to CAI for this special issue. Of those, 12 responded positively. Following a rigorous IJCARS peerreview process by at least three reviewers per paper, ultimately, five papers were selected for inclusion in this issue.

These five papers represent a broad range of emerging topics in CAI. One paper focuses on the outcomes of spine surgery:

- Intraoperative forecasting of growth modulation spine surgery outcomes with spatiotemporal dynamic networks.

$\triangle$ Purang Abolmaesumi

purang@ece.ubc.ca

1 Medical Biophysics, University of Toronto, Toronto, Canada

2 Department of Computer Science, University College London, London, UK

3 LS2N Laboratory, Ecole Centrale Nantes, Nantes, France

4 School of Computer Science, The Hebrew University of Jerusalem, Jerusalem, Israel

5 Department of Electrical and Computer Engineering, The University of British Columbia, Vancouver, Canada
A second paper presents a segmentation method for postoperative MRI following brain surgery:

- A self-supervised learning strategy for postoperative brain cavity segmentation simulating resections.

One paper develops new methods for treatment planning of intracranial aneurysms:

- Intra-saccular device modeling for treatment planning of intracranial aneurysms: from morphology to hemodynamics.

Surgical skill assessment using gesture recognition is the subject of another paper:

- SD-Net: Joint Surgical Gesture Recognition and Skill Assessment.

Finally, one paper explores if audio signals can be used for guiding needle insertions:

- Study of needle punctures into soft tissue through audio and force sensing: Can audio be a simple alternative for needle guidance?

We would like to thank all authors and reviewers who contributed to this IJCARS special issue, and all reviewers and Area Chairs who took part in the MICCAI 2020 conference review process. Special thanks goes to the management of IJCARS, particularly Dr. Heinz Lemke and Franziska Schweikert, who enabled a timely review process for these papers, while ensuring the high quality of this special issue.

Publisher's Note Springer Nature remains neutral with regard to jurisdictional claims in published maps and institutional affiliations. 